\title{
Fingerprint Image Enhancement and Extraction of Minutiae and Orientation
}

\author{
Shancymol Sojan \\ PG Scholar \\ Electronics and Telecommunication Dept \\ VESIT, Mumbai, Maharashtra, India
}

\author{
R. K. Kulkarni, PhD \\ Professor \\ Electronics and Telecommunication Dept \\ VESIT, Mumbai, Maharashtra, India
}

\begin{abstract}
Fingerprints are popular among the biometric - based systems due to ease of acquisition, uniqueness and availability. Fingerprint based biometric systems work by extracting and matching some features on the fingerprint. Due to errors in acquisition phase, it is possible that the scanned fingerprint image is not of a good quality and hence needs to be enhanced before being processed by the feature extracting module. Out of the various features that can be extracted, orientation and minutiae points are the most common ones to be used. This paper discusses some commonly used fingerprint enhancement techniques, the algorithms for minutiae and orientation extraction followed by the comparison of the algorithm on various databases.
\end{abstract}

\section{Keywords}

fingerprints, minutiae, orientation, normalizaiton, spurious minutiae, cross numbering, termination, bifurcation, direction field.

\section{INTRODUCTION}

Authentication and security are one of the major concerns of today's competitive and growing economy. Authentication based on passwords and PINS have a risk of being stolen or misused. In such a case, biometrics acts as an effective solution as it offers high security over these methods. Biometric traits can be either physiological like fingerprint, iris, face, palm or behavioral like gesture, gait etc [1]. Out of these, fingerprints are the most commonly used biometric trait.

There are many features like ridge, core, delta, etc that can be extracted from a finger print [2]. Prior to feature extraction, it is important to enhance the quality of the image to remove unwanted noise which can cause incorrect features being extracted. The fingerprint image enhancement techniques are namely (i) normalization method [3], (ii) histogram equalization by adaptive thresh holding [4], (iii) filtering approach [5], [6] using wiener filter, median filters, Gabor filter, etc.

Minutiae or Galton features are the various ridges and the crossings in the fingerprint. There are many methods available in literature [7]-[20] for extracting minutiae which can broadly be classified into those that work on binarized images and those that work on grey scale images. Methods that work on binarized images can be further classified into thinningbased \& non-thinning based. Examples are chain code based, morphology based, run representation, cross numbering based, etc. Examples of methods that directly work on grayscale images are ridge line following based and fuzzy based. Binarization process sometimes may lead to loss of information and also this process is time consuming. Though it is also possible to detect minutiae from grayscale images directly but is still a topic of research. Thus binarization based methods are preferred and the most commonly used method is cross numbering based method which works on thinned images.

Orientation estimation methods can be categorized as pixelalignment based method, local method and global method [21]. The drawback in pixel- alignment based method is that its accuracy is limited to fixed number of 8 discrete reference orientation values and computational complexity increases proportional to the number of reference orientations. Some of the local methods are filter bank [22], wavelet projection [23], gradient based [24],[25] \& spectral estimation of which the gradient based approach gives a more accurate result. The advantage of gradient based method is that the computed orientations are continuous. However, local methods do not give adequate results when the images are of poor quality. Global methods [26] are based on mathematical modeling of data. Some of the models in literature are zero-pole model, piece-wise linear approximation model, polynomial model etc. Though model based approach is robust to noise, the inefficiency in developing a perfect mathematical model to represent fingerprint of every individual is deterring it from being a popular approach.

The rest of the paper is organized as follows: Section II discusses the fingerprint based biometrics system, various fingerprint features and the image enhancement techniques used for fingerprint. Section III gives the algorithm for minutiae extraction while Section IV gives the algorithm for orientation extraction. Section V gives the results of minutiae and orientation extraction followed by conclusion and future work in section VI.

\section{FINGERPRINT BIOMERTICS SYSTEM}

The steps in fingerprint based biometrics system are (i) capturing fingerprint image (ii) image enhancement (iii) feature extraction (iv) storing the extracted feature (v) matching.

\subsection{Fingerprint Features}

Fingerprints are unique patterns on the epidermis of fingertip which consists of ridges (dark) and valleys (light) on the surface of a finger of an individual. The various features of the fingerprint are shown in Fig 1.They fall into level 1,2 and 3 features. Level 1 features mainly consists of loops, whorls, arches, etc. Level 2 features refer to ridge skeleton and features derived from it. The portions where these ridges break can take the shape of an isolated point, a termination, a bifurcation, etc which are called as minutiae points or Galton details. A good quality fingerprint should have 40 to 100 minutiae points to determine the uniqueness of the fingerprint and to enable matching. Level 3 features include ridge 
contours, core and delta points. Out of all these features, minutiae points are the most commonly used points for fingerprint matching. Another classification of fingerprint features is global and local features [2] where the global features include core area, delta, ridge count, etc and the local features include ridge ending, bifurcation spur, etc.
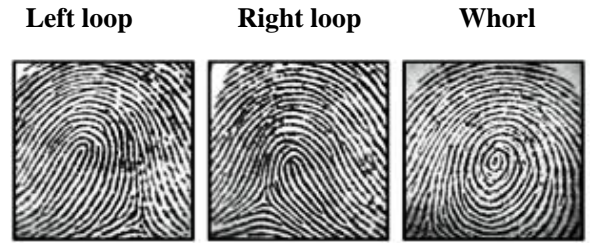

(a)

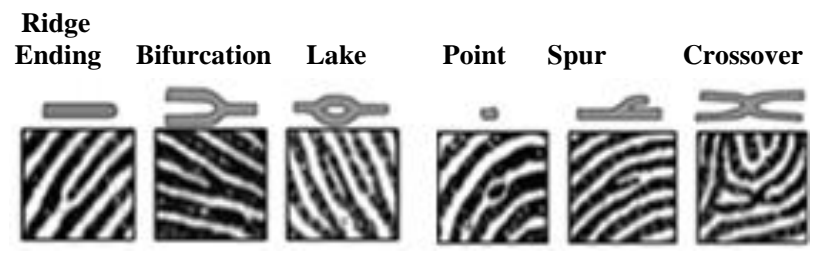

(b)
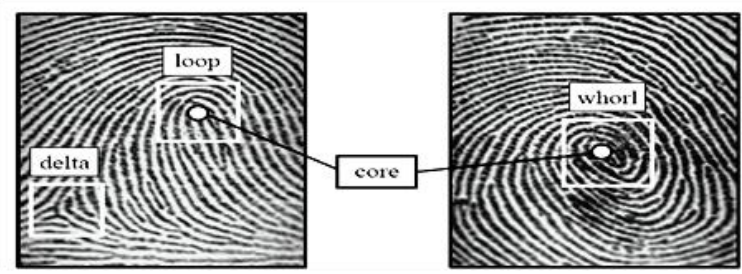

(c)

Fig.1 Fingerprint features (a) Level 1 features (b) Level 2 features(c) Level 3 features [2]

Other than these features, there can be also some false minutiae points due to improper pressure, insufficient ink or due to noise added during scanning. Some of the false minutiae are shown in Fig.2 where (a) is a spike piercing into a valley, (b) is a spike that falsely connects two ridges and (c) has two near bifurcations located in the same ridge.

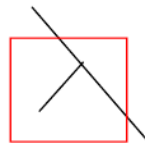

(a)

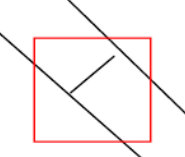

(b)

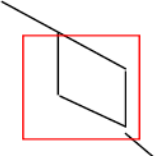

(c)
Fig.2 False minutiae [27]

\subsection{Fingerprint Image Preprocessing}

Fingerprints images captured by sensors may have some ambiguities due to inappropriate finger pressure or due to other noise sources present degrading the quality of fingerprint obtained. This may pose a serious problem in feature extraction and matching processes. Hence some preprocessing has to be done before feature extraction to enhance the quality of the fingerprint. Various methods [3], [4], [5], [6] are available for image enhancement but it depends on the quality of the fingerprint to decide which suits the best.

The fingerprint image pre-processing includes (i) Segmentation -to separate the background and foreground to avoid false minutiae. This is also called ROI extraction. (ii) Image enhancement - to improve the contrast, illumination and quality of image \& (iii) Binarization- to get a clear contrast between ridges (black) and valleys (white).

The image enhancement of fingerprint images can be done in one of the following methods or by using a combination of these methods.

\subsubsection{Histogram Equalization}

The histogram [4] of an image represents the relative frequency of the various gray levels of an image. It is a technique for the improvement in the contrast of an image by adjusting the intensity of every gray level of the image. Histogram equalization with adaptive thresh holding gives a better result.

\subsubsection{Filtering approach}

Various filtering approaches [3], [6], can be used to enhance the quality of fingerprint image. Some of the filters used are wiener filter, median filter, high pass filter, etc which can be used in fingerprints corrupted by noise. Gabor filter is another effective technique as it increases the contrast between the foreground ridges and background.

\subsubsection{Normalization}

Image normalization [5] is one of the commonly used methods for image enhancement. It is pixel wise operation used for intensity correction, the main purpose of which is to reduce the variations in gray-level values along ridges and valleys.

Let $I(i, j)$ denote the gray-level values at pixel $(i, j)$ of dimension $N x N, M$ and $V$ denote the estimated mean and variance of the image respectively, and $N(i, j)$ denote the normalized gray-level value at pixel $(i, j)$. The normalized image is defined as follows:

$$
\begin{aligned}
N(i, j) & =\mathrm{M}_{\mathrm{o}}+\sqrt{\frac{\mathrm{V}_{\mathrm{o}} \cdot(\mathrm{I}(\mathrm{i}, \mathrm{j})-\mathrm{M})^{2}}{\mathrm{~V}}} ; I(i, j)>M \\
= & \mathrm{M}_{\mathrm{o}}-\sqrt{\frac{\mathrm{V}_{\mathrm{o} \cdot(\mathrm{I}(\mathrm{i}, \mathrm{j})-\mathrm{M})^{2}}^{\mathrm{V}}}{\mathrm{V}}} ; \text { otherwise ......... }
\end{aligned}
$$

where $\mathrm{M}_{\mathrm{o}}$ and $\mathrm{V}_{\mathrm{o}}$ are the desired mean and variance values. $\mathrm{M}$ and $\mathrm{V}$ are calculated as follows:

$M=\frac{1}{\mathrm{~N}^{2}} \sum_{\mathrm{i}=0}^{\mathrm{N}-1} \sum_{\mathrm{j}=0}^{\mathrm{N}-1} \mathrm{I}(\mathrm{i}, \mathrm{j})$

$V=\frac{1}{\mathrm{~N}^{2}} \sum_{\mathrm{i}=0}^{\mathrm{N}-1} \sum_{\mathrm{j}=0}^{\mathrm{N}-1}(\mathrm{I}(\mathrm{i}, \mathrm{j})-\mathrm{M})^{2}$

Fig.3 shows the normalized image using the equations (1), (2), (3) with $\mathrm{M}_{\mathrm{o}}=100$ andV $=100$.

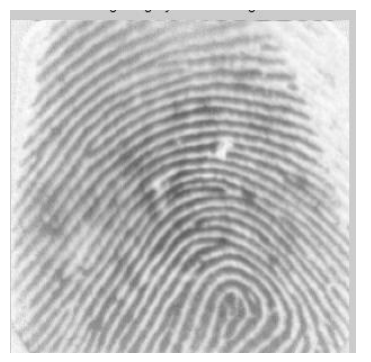

(a)

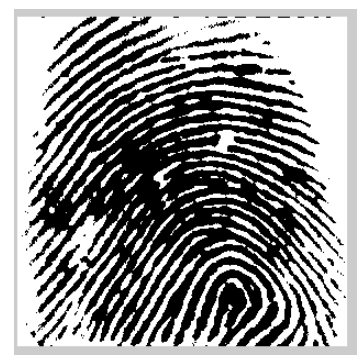

(b)
Fig.3 (a) Input fingerprint (b) Normalized fingerprint 


\section{MINUTIAE EXTRACTION ALGORITHM}

For fingerprint matching, the most widely extracted features are minutiae points. They include:

a. Ridge endings - an abruptly ending ridge.

b. Ridge bifurcation - a ridge that splits into two ridges.

c. Short ridges, island or independent ridge - a ridge travels a short distance and then ends.

d. Ridge enclosures - a single ridge that bifurcates and combines afterwards to continue as a single ridge.

e. Spur - a bifurcation where a short ridge branches off into a longer ridge.

f. Crossover - a short ridge that lies between two parallel ridges [28].

Among these features, ridge terminations (endings) and ridge bifurcations will be used.

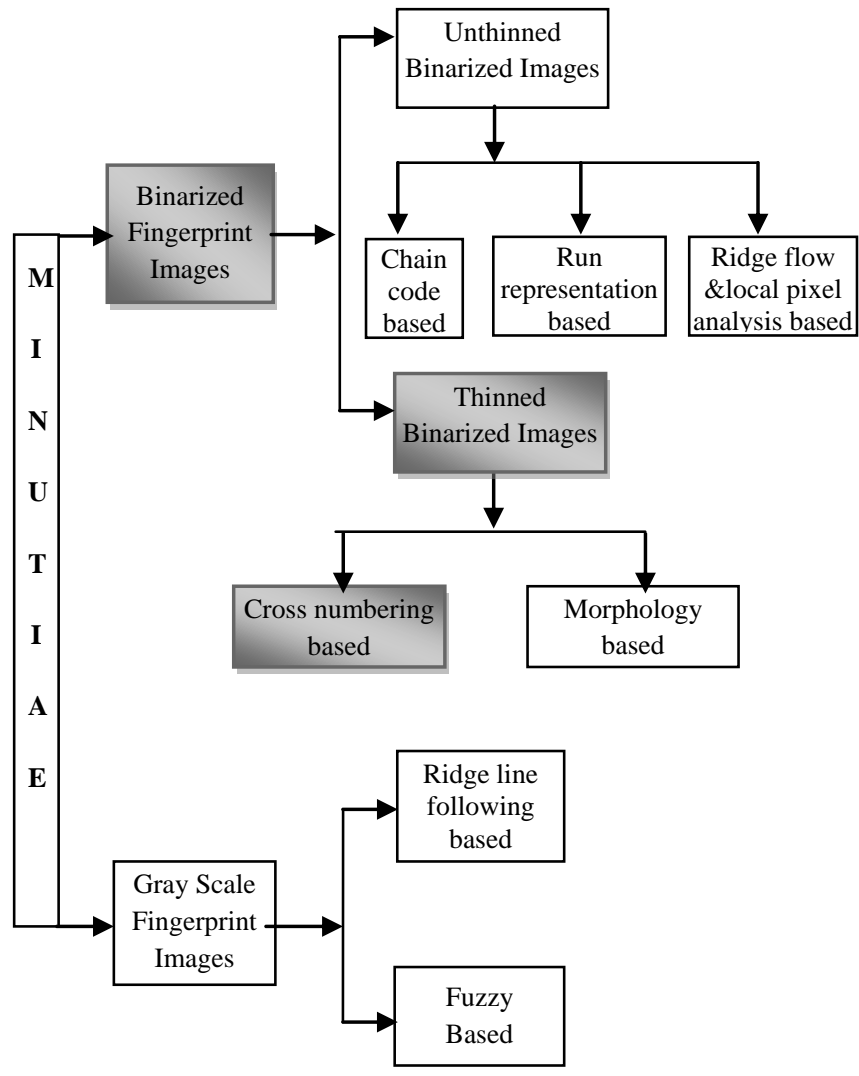

Fig. 4 Minutiae extraction methods [28]

Fig.4 shows the various minutiae extraction methods. The cross numbering concept is the most widely used due to high computational efficiency and simplicity. In this method, 8connected ridge flow pattern of skeletonized image is used and by scanning the local neighbourhood of each pixel, $\mathrm{CN}$ value is computed.

\begin{tabular}{|l|l|l|}
\hline $\mathrm{P}_{4}$ & $\mathrm{P}_{3}$ & $\mathrm{P}_{2}$ \\
\hline $\mathrm{P}_{5}$ & $\mathrm{P}$ & $\mathrm{P}_{1}$ \\
\hline $\mathrm{P}_{6}$ & $\mathrm{P}_{7}$ & $\mathrm{P}_{8}$ \\
\hline
\end{tabular}

Fig.5 Neighbourhood of the pixel in $3 \times 3$ window. [28]

The formula for calculating $\mathrm{CN}$ is as follows:

$\mathrm{CN}=0.5 \sum_{\mathrm{i}=1}^{8}\left|\mathrm{P}_{\mathrm{i}}-\mathrm{P}_{\mathrm{i}+1}\right|$

where $\mathrm{P}_{9}=\mathrm{P}_{1}$. It is defined as half the sum of the differences between pairs of adjacent pixels in the eight-connected neighbourhood. A ridge pixel with a $\mathrm{CN}=1$ corresponds to a ridge ending, and a $\mathrm{CN}=3$ corresponds to a bifurcation. The $3 \times 3$ neighbourhood of the central pixel is shown in Fig.5. The properties of $\mathrm{CN}$ are be summarized in Table 1 .

Table 1. Properties of CN [ 28]

\begin{tabular}{|c|l|}
\hline CN value & Property \\
\hline 0 & Isolated point \\
\hline 1 & Ridge ending point \\
\hline 2 & Continuing ridge point \\
\hline 3 & Bifurcation point \\
\hline 4 & Crossing point \\
\hline
\end{tabular}

The algorithm for extracting minutiae using the concept of cross numbering is summarized in Fig.6.

The algorithm can be divided into the following steps:

(i) Preprocessing or Enhancement:

a) Convert the fingerprint RGB image into gray scale image.

b) Binarizing the image i.e. to convert the gray scale image into binary image by fixing the threshold value. The Matlab function im $2 \mathrm{bw}$ can be used. The threshold chosen is 0.5 .

c) Thinning the image i.e. to reduce the thickness of the ridge lines to a single pixel width. This is done to extract minutiae points effectively. It can be done by bwmorph function in Matlab.

(ii) Minutiae extraction:

For minutiae extraction, first the fingerprint image is divided into many $3 \times 3$ windows and then by using functions minutie and nlfilter, cross number $(\mathrm{CN})$ is computed for each window. For ridge termination, $\mathrm{CN}$ is 1 and for ridge bifurcation $\mathrm{CN}$ is 3. Then these points are marked in different colors to differentiate between them.

(iii)Post processing:

There may be a lot of false ridges which if not removed can affect the accuracy in matching. These can be removed by considering the distance between the minutiae points. If two terminations or two bifurcations or a termination and bifurcation are too close to each other, then they are removed. 


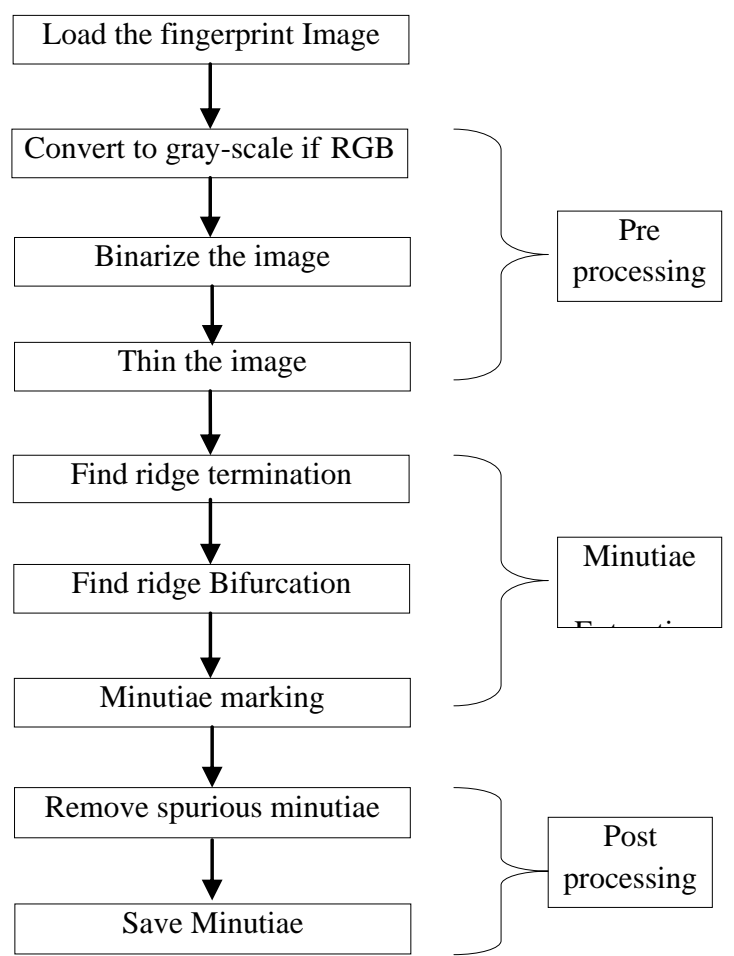

Fig. 6 Flowchart for minutiae extraction

\section{ORIENTATION EXTRACTION ALGORITHM}

The angle formed by the horizontal line with the ridge inclination is called as orientation. It is shown in Fig.7.

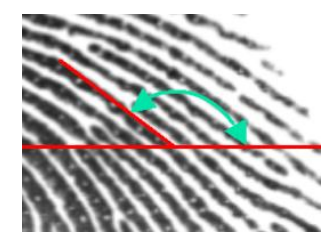

Fig. 7 Orientation [2]

Orientation is computed block-wise and not pixel-wise.Fig. 8 shows the algorithm for orientation estimation of a fingerprint. The procedure can be summarized as follows [24], [25].

\section{(i) Preprocessing or Enhancement:}

(a) Convert the fingerprint RGB image into gray scale image.

(b) Normalize the image. Alternatively histogram equalization can also be used.

\section{(ii) Orientation extraction:}

Orientation or direction is extracted using gradient based approach. The steps are:

(a) Divide the image into $w \times w$ overlapping blocks. The window size is taken as $8 \times 8$.

(b) The gradients Gx and Gy are computed using Sobel filter. Gx represents the horizontal gradient component and Gy represents the vertical gradient component.

(c) The average gradient vectors are computed as follows:

$\overline{\mathrm{Gx}^{\prime}}==\frac{1}{\mathrm{~W} \times \mathrm{W}} \sum_{\mathrm{i}=1}^{\mathrm{W}} \sum_{\mathrm{j}=1}^{\mathrm{W}}\left(\mathrm{Gx}^{2}(\mathrm{i}, \mathrm{j})-\mathrm{Gy}^{2}(\mathrm{i}, \mathrm{j})\right)$

$\overline{\mathrm{Gy}^{\prime}}==\frac{1}{\mathrm{~W} \times \mathrm{W}} \sum_{\mathrm{i}=1}^{\mathrm{W}} \sum_{\mathrm{j}=1}^{\mathrm{W}}(2 \mathrm{Gx}(\mathrm{i}, \mathrm{j}) \mathrm{Gy}(\mathrm{i}, \mathrm{j}))$

(d) Direction Estimation

$$
\begin{aligned}
\theta & =\frac{1}{2} \tan ^{-1} \frac{\overline{\mathrm{Gy}^{\prime}}}{\overline{\mathrm{Gx}^{\prime}}}+\frac{\pi}{2} ; \tan ^{-1} \frac{\overline{\mathrm{Gy}^{\prime}}}{\overline{\mathrm{Gx}^{\prime}}}<0 \\
& =\frac{1}{2} \tan ^{-1} \frac{\overline{\mathrm{Gy}^{\prime}}}{\overline{\mathrm{Gx}^{\prime}}}-\frac{\pi}{2} ; \tan ^{-1} \frac{\overline{\mathrm{Gy}^{\prime}}}{\overline{\mathrm{Gx}^{\prime}}} \geq 0
\end{aligned}
$$

(iii) Plotting:

The direction map is plotted using quiver plot and orientation image is saved.

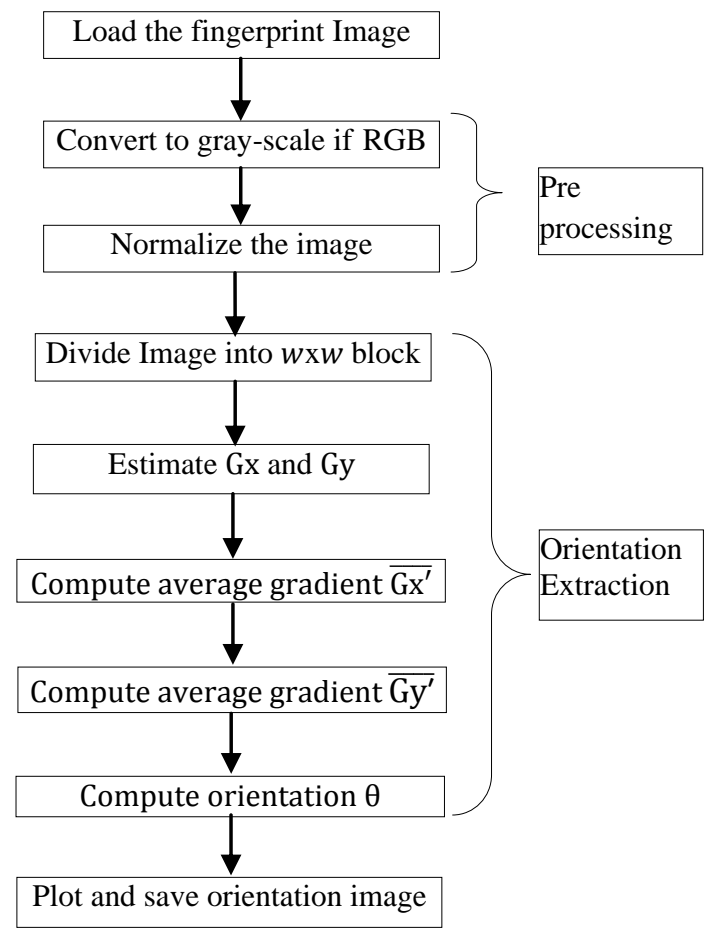

Fig. 8 Flow chart for orientation extraction

\section{RESULTS AND DICSUSSION}

The results of minutiae extraction are shown in Fig.9. Table 2 shows the comparison of minutiae extraction for different images.

The algorithm in Fig.6 was applied to a few images in the standard FVC data set and some random fingerprint images of different sizes. The results are shown in Table 2. It is observed that the run time doesn't depend on the size of the image but on the quality of image and the number of terminations and bifurcations in it. For the NIST database the binarized threshold had to be reduced $(0.41)$ as the illuminations of the image was poor. For all other cases the threshold was taken greater than $50 \%$.The algorithm was also tested on some poor quality images from the database DB2_B. The size of the image is $364 \times 328$. 


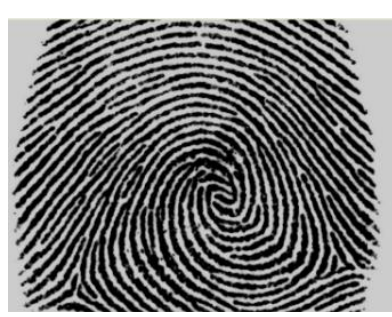

(a)

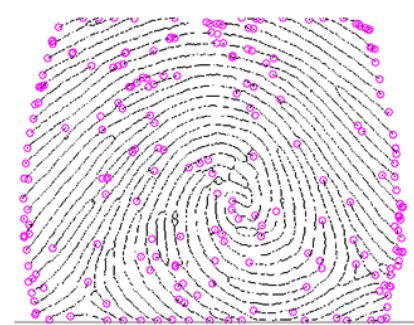

(d)
Pre- Processing

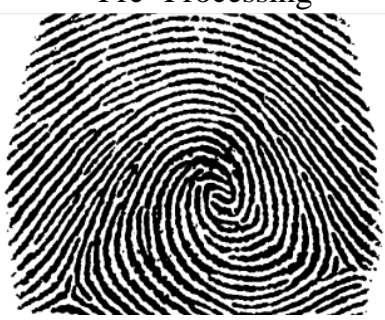

(b)

Minutiae Extraction

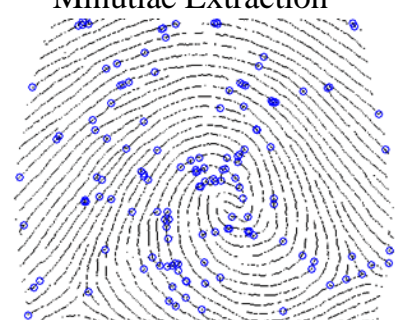

(e)

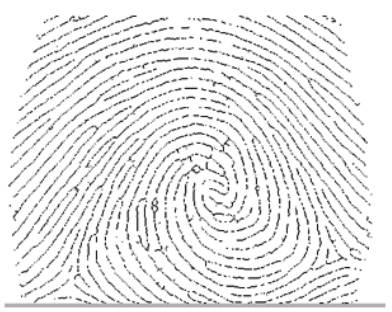

(c)

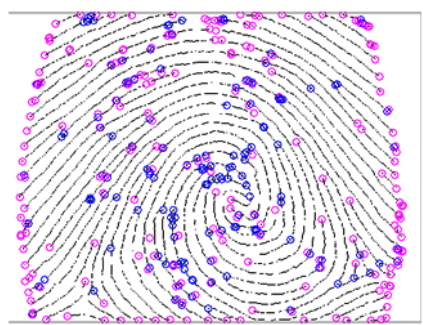

(f)

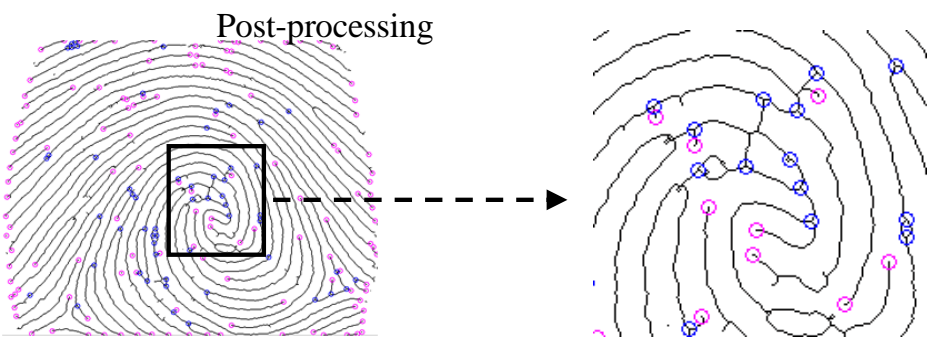

(g)

(h)

Fig 9. (a) input gray-scale fingerprint (b) binarized image (c)thinned image (d) terminations (e) bifurcations (f) all detected minutiae points (g) after removing spurious minutiae (h) enlarged cross-section of minutiae.

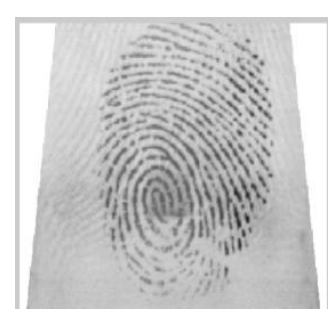

(a)

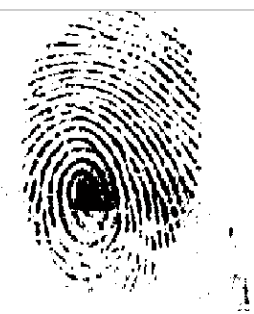

(b)

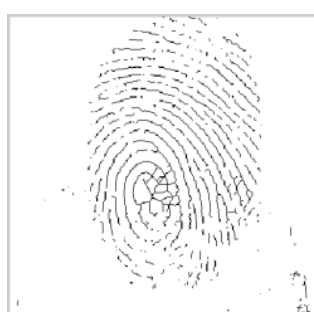

(c)

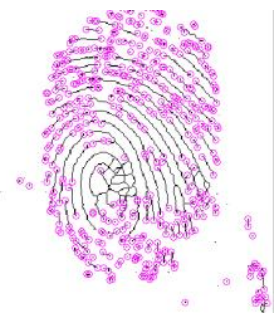

(d)

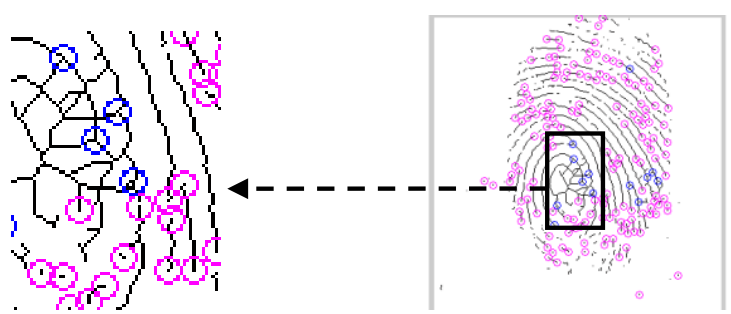

(h)

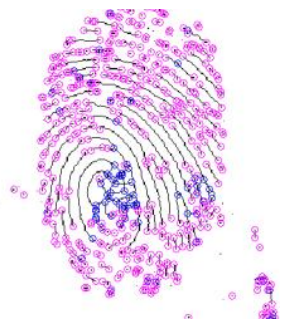

(f)

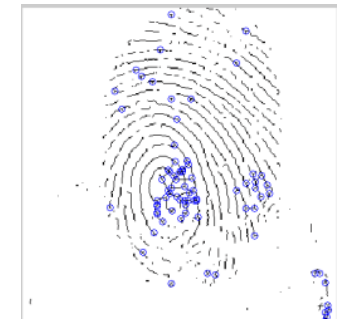

(e)

Fig 10. (a) input gray-scale fingerprint (b) binarized image (c)thinned image (d) terminations (e) bifurcations (f) all detected minutiae points (g) after removing spurious minutiae (h) enlarged cross-section of minutiae after removing spurious minutiae (h) enlarged cross-section of minutiae 
Pre- Processing by adaptive histogram equalization

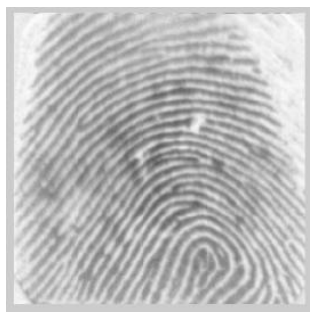

(a)

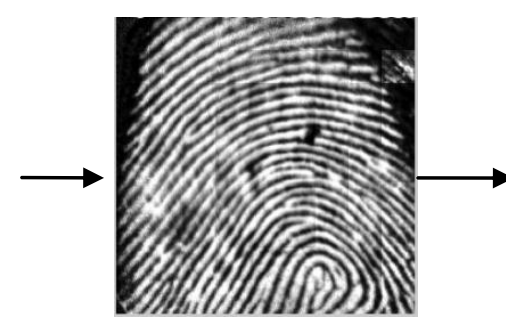

(b)

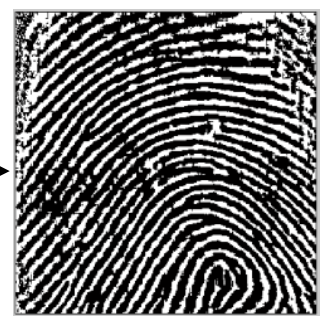

(c)

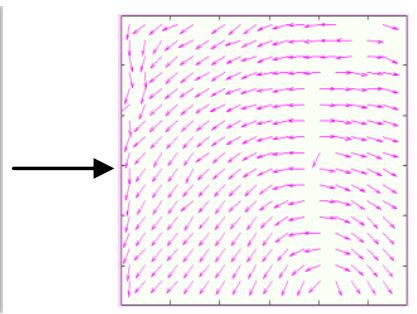

(d)

Fig 11. (a) input fingerprint, (b) histogram equalized image, (c) adaptive thresholding, (d) orientation image

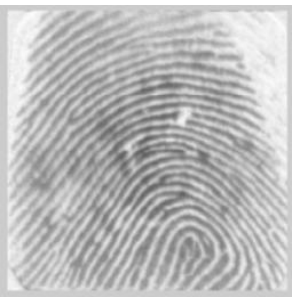

(a) Pre- Processing by Normalization

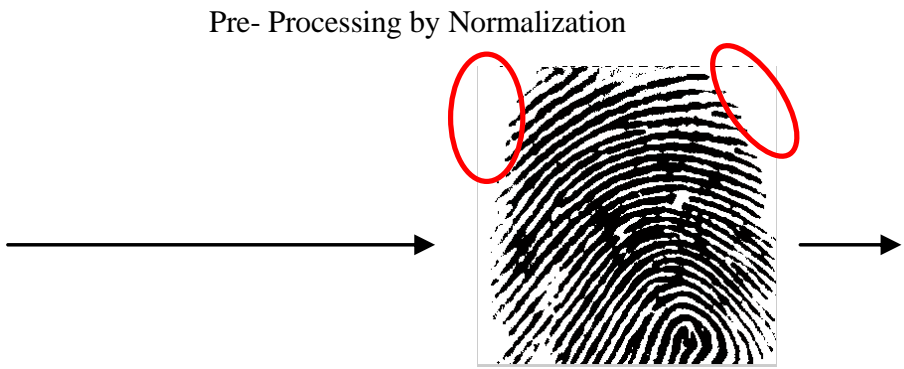

(b)

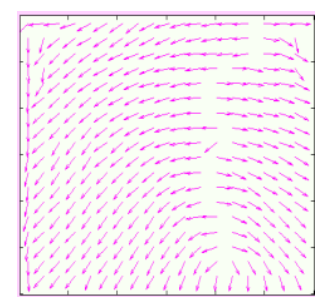

(c)

Fig 12. (a) input fingerprint, (b) normalized image, (c) orientation image

As can be seen in Fig.10 (a) the image is of a poor with lot of background noise. The binarization process in (b) helps to clearly separate the fingerprint from the background. Here the threshold chosen is 0.65 . The minutiae points extracted are seen in (d),(e),(f). As can be seen there are lot of false minutiae, but they are removed after post-processing as can be seen in $(\mathrm{g})$. Around 163 terminations and 13 bifurcations were detected. As can be seen from Table 1, the algorithm works fine on different databases and different size of images. Also by changing the binarization threshold, it can work effectively on poor quality images. There are a lot of detected minutiae points, all of which may not be used in matching as it will be time consuming. So it is possible to separate a few points which can be used in matching.

The results of orientation extraction are shown in Fig.11 and Fig.12.The size of images was $300 \times 300$ pixels taken from FVC 2000 dataset. Fig.11 shows pre-processing by adaptive histogram equalization and Fig.121 shows pre-processing by normalization approach. As can be seen in Fig.11 (b),(c) adaptive histogram equalization has added some noise around the boundaries as compared to normalization in Fig.12 (b). The run time or both methods are almost same. It can be concluded that normalization gives a better estimate of the enhanced fingerprint can be a preferred method for extraction of orientation.

Table 2. Minutiae Extraction

\begin{tabular}{|c|c|c|c|c|c|}
\hline Database & & $\begin{array}{l}\text { Image size } \\
\text { (pixels) }\end{array}$ & $\begin{array}{l}\text { No. of of } \\
\text { terminatio } \\
\text { ns }\end{array}$ & $\begin{array}{l}\text { No. of } \\
\text { bifurcation } \\
\text { s }\end{array}$ & $\begin{array}{l}\text { Run time } \\
\text { (secs) }\end{array}$ \\
\hline \begin{tabular}{|l} 
FVC \\
DB1_B \\
\end{tabular} & 2000 & $350 \times 306$ & 68 & 142 & 6.496990 \\
\hline \begin{tabular}{|l} 
FVC \\
DB2_B
\end{tabular} & 2000 & $292 \times 286$ & 82 & 138 & 5.392366 \\
\hline $\begin{array}{l}\text { FVC } \\
\text { DB3_B }\end{array}$ & 2000 & $400 \times 400$ & 37 & 113 & 4.755139 \\
\hline FVC & 2000 & $384 \times 288$ & 160 & 1 & 5.193061 \\
\hline
\end{tabular}

\begin{tabular}{|l|c|c|c|c|}
\hline DB4_B & & & & \\
\hline Nist_sd_04 & $512 \times 512$ & 113 & 242 & 5.244311 \\
\hline Random image & $520 \times 700$ & 130 & 56 & 5.774122 \\
\hline
\end{tabular}

6. CONCLUSION AND FUTURE WORK

Fingerprint based biometrics systems are the most commonly used biometrics systems. Image enhancement and feature extraction are the core of this system. This paper has presented a review on various finger print image enhancement techniques, minutiae extraction methods and orientation extraction methods. Minutiae extraction by cross numbering concept and orientation extraction by gradient based method have been implemented.

Future work in feature extraction can be to crop a region of interest and then extract features from that region to save the computational time or to use patches in the fingerprint to process the minutiae. Better image enhancement methods can be used to improve the performance of the system if the image quality is poor.

\section{REFERENCES}

[1] Delac, Kresimir, and Mislav Grgic. "A survey of biometric recognition methods." Electronics in Marine, 2004. Proceedings Elmar 2004. 46th International Symposium. IEEE, 2004.

[2] Maltoni, Davide, et al. Handbook of fingerprint recognition. Springer Science \& Business Media, 2009

[3] Thai, Raymond. "Fingerprint image enhancement and minutiae extraction."The University of Western Australia (2003).

[4] Hanoon, Muna F. "Contrast fingerprint enhancement based on histogram equalization followed by bit reduction of vector quantization." International Journal of Computer Science and Network Security 11.5 (2011): 116-123. 
[5] Hong, Lin, Yifei Wan, and Anil Jain. "Fingerprint image enhancement: algorithm and performance evaluation." Pattern Analysis and Machine Intelligence, IEEE Transactions on 20.8 (1998): 777-789.

[6] Greenberg, Shlomo, et al. "Fingerprint image enhancement using filtering techniques." Pattern Recognition, 2000. Proceedings. 15 $5^{\text {th }}$ International Conference on. Vol. 3. IEEE, 2000.

[7] Carneiro, Romulo Ferrer L., et al. "Techniques of Binarization, Thinning and Feature Extraction Applied to a Fingerprint System." International Journal of Computer Applications 103.10 (2014).

[8] Zhixin Shi, Venu Govindaraju, "A chaincode based scheme for fingerprint feature extraction", Pattern Recognition Letters, vol. 27, 2006, pp. 462-468

[9] Zenzo, L. Cinque, and S. Levialdi, "Run-Based Algorithms for Binary Image Analysis and Processing", IEEE Trans. Pattern Analysis and Machine Intelligence, vol. 18, no. 1, 1996, pp. 83-88.

[10] J Hwan Shin, H. Y. Hwang, S Chien, "Detecting fingerprint minutiae by run length encoding scheme", Pattern Recognition vol. 39, 2005, pp. 1140-1154.

[11] S. Maddala, S. R. Tangellapally, J. S. Bartuněk and M. Nilsson, "Implementation and evaluation of NIST Biometric Image Software for fingerprint recognition," Biosignals and Biorobotics Conference (BRC), 2011 ISSNIP, pp.1-5.

[12] M. Gamassi, V. Pivri and F. Scotti, "Fingerprint local analysis for high performance minutiae extraction", IEEE International Conference on Image Processing (ICIP) vol. 3, 2005, pp. 265-268.

[13] E. Alibeigi, M. T. Rizi, P. Behnamfar, "Pipelined minutiae extraction from fingerprint images," Electrical and Computer Engineering, 2009. CCECE '09. Canadian Conference on , vol., no., pp.239-242.

[14] J. C. Amengual, A. Juan, J. C. Prez, F. Prat, S. Sez, and J. M. Vilar, "Real-time minutiae extraction in fingerprint images", in Proc. of the 6th Int. Conf. on Image Processing and its Applications, 1997, pp. 871-875.

[15] A. Farina, Z. M. Kovacs-Vajna, and A. Leone, "Fingerprint minutiae extraction from skeletonized binary images", Pattern Recognition, vol. 32(5), 1999, pp. 877-889.

[16] J. Xudong and Y. Wei-Yun, "Fingerprint minutiae matching based on the local and global structures", in Proc. of International Conference on Pattern Recognition (ICPR), vol. 2, 2000, pp. 1038-1041

[17] R. Bansal, P. Sehgal, P. Bedi, "Effective Morphological Extraction of True Fingerprint Minutiae based on the Hit or Miss Transform", International Journal of Biometrics and Bioinformatics(IJBB), vol. 4, 2010, pp. 71-85.

[18] L. Jinxiang, H. Zhongyang, and C. Kap Luk, "Direct minutiae extraction from gray-level fingerprint image by relationship examination", in International Conference on Image Processing(ICIP), vol. 2, 2000, pp. 427-430.

[19] X. Jiang, W.-Y. Yau, and W. Ser, "Detecting the fingerprint minutiae by adaptive tracing the gray-level ridge", Pattern Recognition, vol. 34(5), 2001, 999-1013

[20] V. K. Sagar, D. B. L. Ngo, and K. C. K. Foo, "Fuzzy feature selection for fingerprint identification", in proc. 29th Annual International Carnahan.Security Technology, 1995, pp 85-90.

[21] Hou, Zujun, Wei-Yun Yau, and Yue Wang. "A review on fingerprint orientation estimation." Security and Communication Networks 4.5 (2011): 591-599.

[22] Biradar, Vidaydevi G., and H. Sarojadevi. "Fingerprint Ridge Orientation Extraction: A Review of State of the Art Techniques." International Journal of Computer Applications 91.3 (2014).

[23] Wieclaw, Lukasz. "Fingerprint Orientation Field Enhancement." Computer Recognition Systems 4. Springer Berlin Heidelberg, 2011. 33-40.

[24] WIECLAW, Lukasz. "Gradient based fingerprint orientation field estimation."Journal of Medical Informatics \& Technologies 22 (2013): 203-207.

[25] Ke, Hongchang, Hui Wang, and Degang Kong. "An improved Gabor filtering for fingerprint image enhancement technology." 2nd

[26] Zhou, Jie, and Jinwei Gu. "A model-based method for the computation of fingerprints' orientation field." Image Processing, IEEE Transactions on 13.6 (2004): 821-835.

[27] Barham, Zain S., and Allam Mousa. "Fingerprint Recognition using MATLAB." (2011).

[28] Bansal, Roli, Priti Sehgal, and Punam Bedi. "Minutiae extraction from fingerprint images-A Review." arXiv preprint arXiv:1201.1422 (2011) 